\title{
A BEAT FREQUENCY RF MODULATOR FOR GENERATION OF LOW REPETITION RATE ELECTRON MICROBUNCHES FOR THE CEBAF POLARIZED SOURCE*
}

\author{
J. Musson\#, J. Grames, J. Hansknecht, R. Kazimi, M. Poelker Jefferson Laboratory, Newport News, \\ Virginia 23606, USA
}

\begin{abstract}
Fiber-based drive lasers now produce all of the spinpolarized electron beams at CEBAF/Jefferson Lab. The flexibility of these drive lasers, combined with the existing three-beam CEBAF photoinjector Chopper, provides a means to implement a beat frequency technique to produce long time intervals between individual electron microbunches (tens of nanoseconds) by merely varying the nominal $499 \mathrm{MHz}$ drive laser frequency by $<20 \%$. This submission describes the RF Laser modulator that uses a divider and heterodyne scheme to maintain coherence with the accelerator Master Oscillator (MO), while providing delay resolution in increments of $2 \mathrm{~ns}$. Some possible uses for such a beam are discussed as well as intended future development.
\end{abstract}

\section{INTRODUCTION}

Under normal operating conditions, the CEBAF photoinjector generates three interleaved electron beams, each having a repetition rate of $499 \mathrm{MHz}$, the third subharmonic of the acceleration cavities. This unique structure is achieved by using three individual photogun drive lasers to produce 40 ps bunches with 2 ns spacing. In $\mathrm{CW}$ mode, the drive lasers are synchronous with the rest of the machine, producing electron bunches that fill every RF period of the accelerator.

The CEBAF photoinjector chopper system sets the phase acceptance of the injector. It employs two $499 \mathrm{MHz}$ TM210 cavities to deflect and recombine the electron beam after it passes through slits on a water-cooled copper mask. Only electrons within a 111 ps window (20 degrees at $499 \mathrm{MHz}$ ) pass through the mask. The chopping process is also synchronous with the accelerating cavities [1], [2].

Recent parity-violation experiments have requested longer bunch spacings to facilitate the separation of high-energy, elastically-scattered protons from lower-energy particles [3]. Early CEBAF injector configurations employed a separate $31 \mathrm{MHz}\left(16^{\text {th }}\right.$ subharmonic) mode-locked laser, resulting in $32 \mathrm{~ns}$ bunch spacing. Many hours were required to install and remove this dedicated laser system, and any attempts to automate the change were dismissed.

Although lower subharmonic drive laser frequencies appear attractive, it is difficult to maintain pulse symmetry and consistency for $100 \mathrm{MHz}$ repetition rates and below. In addition, the optical cavity size for low repetition rates becomes large and intractable. An alternative solution involves unlocking the drive laser RF, and using the chopper as an analyzer, resulting in the creation of a heterodyne, or beat frequency, after the chopper mask [4]. This technique then allows the gun to continue to produce uniform bunches at near- $499 \mathrm{MHz}$ repetition rates, while the chopper output only contains bunches as defined by the beat frequency.

Concurrent with the recent run of the Hall $\mathrm{C} \mathrm{G}_{0}$ experiment, the JLab Electron Gun Group was evaluating the utility of high-power fiber lasers, which are prevalent in the telecommunications industry. The merits of these lasers are high peak power, relatively fast modulation speeds, and reliability. The fiber lasers are mounted in an accessible chassis, whereby optical fiber transmits the light to the tunnel, and ultimately to the photocathode. Most importantly, these lasers produce picosecond pulses via gain switching, a purely electrical technique that does not require changes to the laser cavity length or complicated feedback loops. And because the pulse repetition rate from a gain switched fiber based laser can be varied by simply changing the applied RF signal, these lasers are well suited for implementing the beat frequency technique.

\section{MODULATION}

Only certain frequencies applied to the fiber-based drive lasers will produce a periodic pulsetrain downstream of the chopper. Determination of appropriate frequencies for a given bunch-spacing requirement follows the equation:

$$
\begin{aligned}
& T_{\text {bunch }}= \frac{1}{F_{\text {chopper }}-F_{\text {laser }}}=\frac{N}{F_{\text {chopper }}} \\
& T_{\text {bunch }}=\text { interval between bunches, seconds } \\
& F_{\text {chopper }}=\text { Chopper RF frequency, } \mathrm{MHz} \\
& F_{\text {laser }}=\text { Laser RF frequency, MHz }
\end{aligned}
$$

which yields

$$
F_{\text {laser }}=\left(1-\frac{1}{N}\right) \times F_{\text {chopper }}
$$

\footnotetext{
"Notice: Authored by Jefferson Science Associates, LLC under U.S. DOE Contract No. DE-AC05-06OR23177. The U.S. Government retains a nonexclusive, paid-up, irrevocable, world-wide license to publish or reproduce this manuscript for U.S. Government purposes. 
$\mathrm{N}$ is an integer $>1$, and is equivalent to saying every $\mathrm{Nth}$ RF bucket contains an electron bunch.

A listing of parameters to achieve the demonstrated bunch spacings, with a Chopper frequency of $499 \mathrm{MHz}$, is given in Table 1.

Table 1: Selected laser drive frequencies for given bunch spacings and corresponding values of $\mathrm{N}$.

\begin{tabular}{|l|l|l|l|}
\hline $\begin{array}{l}\text { Bunch Spacing } \\
(\mathrm{nsec})\end{array}$ & $\mathrm{N}$ & $\begin{array}{l}\text { Laser Frequency } \\
(\mathrm{MHz})\end{array}$ & $\begin{array}{l}\text { Bunch Frequency } \\
(\mathrm{MHz})\end{array}$ \\
\hline 10.0 & 5 & 399.2 & 99.8 \\
\hline 16.0 & 8 & 436.625 & 62.375 \\
\hline 20.0 & 10 & 449.10 & 49.9 \\
\hline 32.1 & 16 & 467.8125 & 31.1875 \\
\hline 40.1 & 20 & 474.05 & 24.95 \\
\hline 50.1 & 25 & 479.04 & 19.96 \\
\hline
\end{tabular}

Note that frequencies derived from many of the $\mathrm{N}$-integer values result in repeating decimals. This is problematic when using a separate synthesizer having finite dividers. It causes the bunches to precess around the mask, thereby creating slow beam intensity modulations.

\section{Initial Tests}

Initial proof-of-concept experiments at $31.1875 \mathrm{MHz}$ involved a HP8662 synthesized RF source as the drive laser signal, synchronized to the $10 \mathrm{MHz}$ Master Oscillator. Although bunches possessing the proper spacing were produced, excessive tails and asymmetries were observed by the incoherence of the beat frequency as a result of absence of true phase lock of the synthesizer time base reference to the $\mathrm{MO}$, as well as residual phase noise within the synthesizer. Only those values of $\mathrm{N}$ resulting in laser frequencies of less than 5 decimals were investigated, due to the repeating decimal problem. A viewer located at the Chopper mask was used to qualitatively examine the resulting $\mathrm{N}-1$ spots on the mask, as shown in Figure 1. Although the first experiments provided proof-of-principle, the resulting transported beam quality was not acceptable to the users.

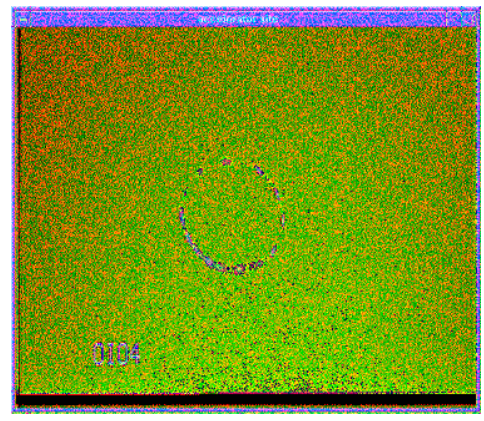

Figure 1: Chopper mask viewer for $40 \mathrm{uA}$ beam, N=16 $32 \mathrm{~ns}$ delay, showing the creation of 15 (N-1) microbunches.
Irregularities and tails are evident due to lack of true phase lock within the HP8662.

\section{Modulator Development}

A dedicated $\mathrm{G}_{0}$-beam modulator was constructed, requiring only $499 \mathrm{MHz}$ input from the Low-Level RF Module (LLRF) as a reference. This topology, using a combination of frequency division and heterodyning, was inherently synchronous to the rest of the accelerator. The modulator produced a $32 \mathrm{~ns}$ bunch spacing, without introducing drift or excessive phase noise. The schematic is shown in Figure 2.

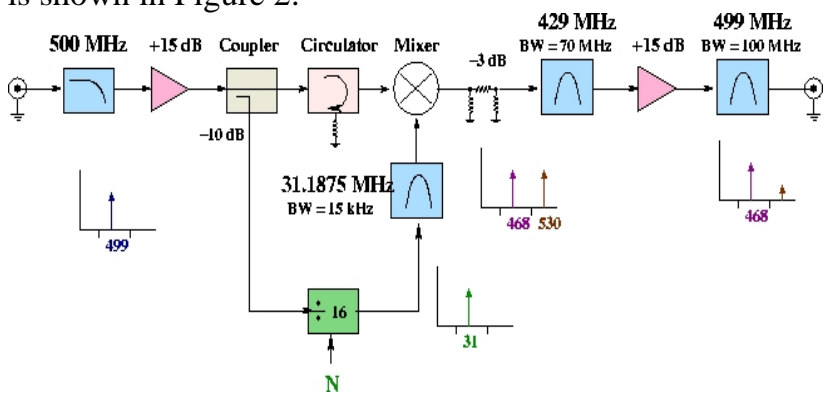

Figure 2: Schematic diagram of Beat-frequency laser modulator and related spectra. Divider and filtering are selected for required bunch spacing.

Results of the modulator provided better uniformity of bunches, especially when aided by the application of electron pre-bunching. User-quality beam with $32 \mathrm{~ns}$ spacing was successfully delivered to the experimental halls although modest residual low frequency intensity modulation was present (Figure 3), a result of inadequate output filtering of the $436 \mathrm{MHz}$ sideband produced from LO leakage on the mixer.

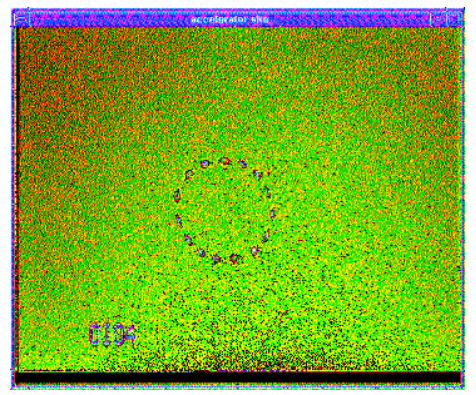

Figure 3: Chopper mask viewer showing modulator-produced beam. Better uniformity is observed, but a low-frequency component is still present. Beam current $=40 \mathrm{uA}$.

A second modulator was built using an Analog Devices AD9511 Clock Generator IC evaluation board. Features of this IC include extremely low additional jitter and phase noise, multiple USB-programmable dividers, choice of logic families, and software-variable output delay [5]. The primary benefit of the AD9511 is to minimize reconfiguration time, and provide computer control. In addition, IF and output filtering were optimized to reduce the unwanted sidebands to improve bunch consistency, as well as provide reasonable flexibility for other values of 
N. The beam quality with 32 ns bunch spacing was improved considerably, as indicated by the chopper slit scan in Figure 4, comparing results with new and old modulator. Clean beat frequency beam is indicated by large contrast between peaks and valleys and an indication of good isolation between consecutive beatbeam pulses.

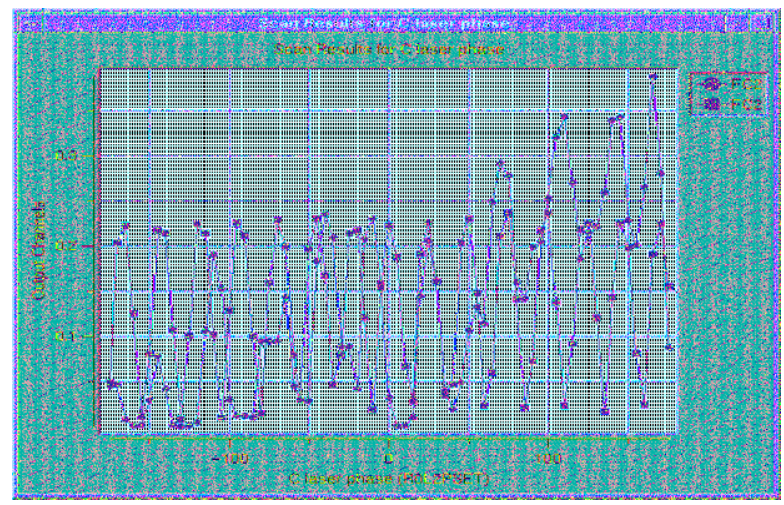

Figure 4: Chopper mask slit scan of modulator-produced beam, and related scan. Better uniformity is observed, but a lowfrequency component is still present. Beam current $=40 \mathrm{uA}$.

As a demonstration of flexibility, rapid configuration changes were made to $40 \mathrm{uA} \mathrm{CW}$ beam for $\mathrm{N}=5,6,8$, and 16 , requiring only minutes to complete. Figure 5 demonstrates beam spacings of $10 \mathrm{~ns}$ and $12 \mathrm{~ns},(\mathrm{~N}=5$ and 6 , respectively). The $12 \mathrm{~ns}$ condition results in a repeatingdecimal drive frequency of $415.833 \ldots \mathrm{MHz}$, and is easily handled by the modulator.

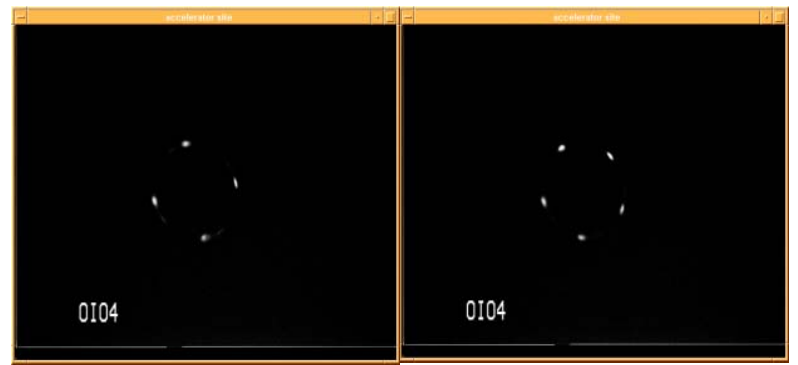

Figure 5: Chopper mask views of $10 \mathrm{~ns}(\mathrm{~N}=5)$ and $12 \mathrm{~ns}(\mathrm{~N}=6)$ bunch spacings, I=40 uA. Demonstrated uniformity, ease of configuration, and ability to create run-on decimal frequencies.

\section{CONCLUSION}

Already, many experimenters have requested this new CEBAF beam structure, for example, to identify sources of background, detector calibration and polarimeter systematic studies. Figure 6 demonstrates clear pion and electron separation at the Hall C Cerenkov detector, data that could not have been obtained using normal CEBAF beam with $2 \mathrm{~ns}$ bunch spacing [6]. The versatile CEBAF photoinjector, including the Chopper and fiber laser systems, provides this capability. The new modulator described above has demonstrated synchronous beam production possessing $2 * \mathrm{~N}$ ns bucket delay times for any $\mathrm{N}$, using only $499 \mathrm{MHz}$ as a reference. Beat frequency beam is clean, stable and adequate for many user applications. Future modulator boards will utilize bandswitched filters, as well as comb filtering, facilitated by the AD9511 variable-delay output.

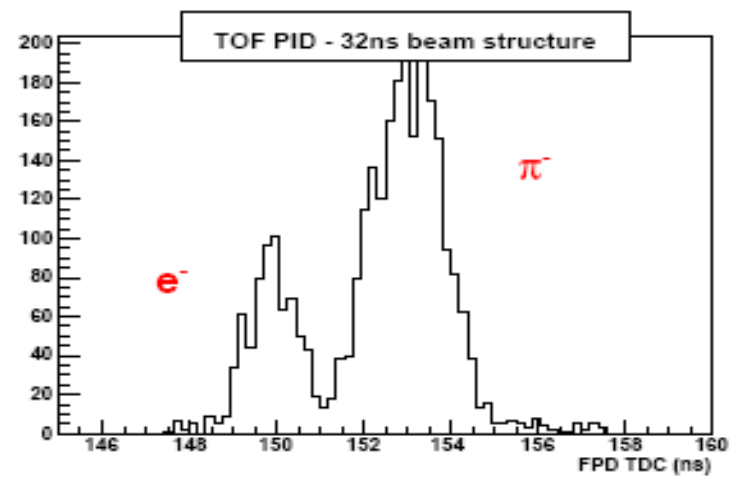

Figure 6: Electron-pion spectrum, demonstrating utility and user-quality of beat-frequency beam.

\section{REFERENCES}

1. R. Abbott, S. Benson, M. Crofford, D. Douglas, R. Gonzales, R. Kazimi, D. Kehne, G.A. Krafft, P. Liger, H. Liu, D. Machie and C. Sinclair, Proceedings of the 1994 International LINAC Conference, Tsukuba, Japan, Aug. 21 - 26, 1994, edited by K. Takata, Y. Yamzaki, K. Nakahara.

2. C. K. Sinclair, et al., Phys. Rev. ST Accel. Beams 10, 023501 (2007).

3. D.S. Armstrong, et al., Phys. Rev. Lett., 95, 092001 (2005)

4. M. Poelker, et al. "Generation of Electron Bunches at Low Repetition Rates Using a BeatFrequency Technique," Phys. Rev. ST Accel. Beams, 10, 053502 (2007)

5. AD9511 Data Sheet, Analog Devices, Inc. http://www.analog.com

6. $G^{0}$ Backward Angle Measurement at Hall C, CEBAF experiment designation E04-115 http://www.jlab.org/div_dept/physics_division/ 